\title{
The Occurrence of Metabolic Risk Factors Stratified by Psoriasis Severity: A Swedish Population-Based Matched Cohort Study
}

This article was published in the following Dove Press journal: Clinical Epidemiology

\author{
Mohammadhossein \\ Hajiebrahimi (iD ${ }^{1,2}$ \\ Ci Song (iD) ${ }^{3}$ \\ David Hägg (iD) ' \\ Therese M-L Andersson (iD) ${ }^{4}$ \\ Reginald Villacorta ${ }^{5}$ \\ Marie Linder (ID) \\ 'Centre for Pharmacoepidemiology, \\ Department of Medicine, Karolinska \\ Institutet, Stockholm, Sweden; \\ ${ }^{2}$ Department of Statistics and \\ Epidemiology, Public Health Faculty, \\ Golestan University of Medical Sciences, \\ Gorgan, Iran; ${ }^{3}$ Janssen GCSO, Stockholm, \\ Sweden; ${ }^{4}$ Department of Medical \\ Epidemiology and Biostatistics, \\ Karolinska Institutet, Stockholm, Sweden; \\ ${ }^{5}$ Market Access Department, Janssen \\ Pharmaceuticals, Horsham, PA, USA
}

Purpose: To assess the relative risk of metabolic risk factors in patients stratified by psoriasis severity compared with population controls.

Patients and Methods: A retrospective cohort study was conducted using national Swedish registers. Adult patients with psoriasis were selected if they had a dispensing of anti-psoriasis prescription (2007-2013) and at least one diagnosis within five years before the dispensing date. The patients with psoriasis were matched 1:10 to controls from the general population on birth year, sex, and county. The cohort was further divided into three disease severity groups (mild, moderate, or severe) based on their dispensed anti-psoriasis medication. Subjects were followed from the index date until censoring. We applied flexible parametric modeling to understand the risks of the incident comorbidities hypertension, hypercholesterolemia, and diabetes mellitus among patients with psoriasis from 6 months through 10 years. Hazard ratios and predicted risk (ie, 1 minus the survival probability) of comorbidities were reported.

Results: The hazard of hypertension, hypercholesterolemia, and diabetes mellitus is higher among psoriasis patients compared with population controls, and the hazard ratio increases with psoriasis severity. For example, HRs of hypertension for patients with mild, moderate, and severe psoriasis are 1.29 (95\% CI: 1.27-1.32), 1.35 (95\% CI: 1.32-1.38), and 1.73 (95\% CI: 1.64-1.82), respectively. The predicted risk of hypertension, hypercholesterolemia, and diabetes mellitus among patients with severe psoriasis at year ten was 0.58 (95\% CI: $0.56,0.59), 0.33$ (95\% CI: $0.32,0.35)$, and 0.21 (95\% CI: $0.20,0.23)$, respectively, while it was $0.42(0.41,0.43), 0.23(0.22,0.23), 0.11(0.10$, 0.11 ) among controls, respectively. The predicted risk at year ten was similar among patients with mild or moderate psoriasis.

Conclusion: The HRs and predicted risks of metabolic risk factors are higher among patients with psoriasis compared with matched controls and are more prominent among the severe psoriasis group.

Keywords: psoriasis severity, predicted risk, survival probability, population-based, metabolic risk factors

\section{Introduction}

Psoriasis is a chronic immune-mediated inflammatory disease which can affect up to $8 \%$ of the population in Nordic countries. ${ }^{1,2}$ Patients with psoriasis experience an increased risk of developing metabolic risk factors such as diabetes mellitus (DM), hypertension (HTN), or hypercholesterolemia. ${ }^{3-12}$ Through a hospital-based study,
Correspondence: Mohammadhossein Hajiebrahimi

Email mohammadhossein.

hajiebrahimi@synergusrwe.com 
Sommer et $\mathrm{al}^{12}$ have reported odds ratios of 2.48, 3.27, and 2.09 for diabetes mellitus, hypertension, and hypercholesterolemia, respectively, among patients with psoriasis compared to patients without psoriasis admitted to the same hospital. Further, previous studies suggest associations between cardiovascular diseases (CVD) and psoriasis. $^{5,7,8,10,13}$ However, few studies examine the incidence of metabolic risk factors as measured by the use of prescription medicines for diabetes mellitus, hypertension, and hypercholesterolemia. For example, Lee et $\mathrm{al}^{14}$ have found the incidence of diabetes mellitus (type 2) 18.2 per 1000 person-years in patients with severe psoriasis while the incidence was 10.5 in mild psoriasis and 5.4 in the comparison cohort consisting of a random sample of patients without a diagnosis of psoriasis from the same patient dataset. Some studies also suggest that treatments for psoriasis could control the inflammatory conditions and alter the metabolic risk factors in patients with psoriasis. $^{15-17}$

Understanding whether patients with psoriasis are being actively managed regarding metabolic risk factors is a research gap in the literature. ${ }^{18}$ Based on prior work that demonstrates an increased risk of hypertension and diabetes mellitus among Swedish patients with severe psoriasis during a one-year period before anti-psoriasis drug prescription, ${ }^{19}$ this study aims to understand the time to occurrence of metabolic risk factors such as diabetes mellitus, hypertension, and hypercholesterolemia. The objective of this study was to assess the relative risk of metabolic risk factors in patients stratified by psoriasis severity compared with population controls.

\section{Patients and Methods}

\section{Data Source and Study Design}

This was a retrospective cohort study using national registers in Sweden. The source data consist of a matched cohort study of patients with psoriasis, selecting up to ten controls randomly from the general population matched on birth year, sex, and county where they are living at the first psoriasis diagnosis and alive and without a record of psoriasis at the first psoriasis diagnosis of the corresponding patient. The study source population has been described in more detail elsewhere, ${ }^{19}$ but in summary, the Swedish National Patient register (NPR) has national coverage for inpatient hospitalizations since 1987 and for outpatient visits since $2001 .^{20}$ The Cause of Death Register (CDR) records dates as well as causes of death. ${ }^{21}$
The Prescribed Drug Register (PDR) records all dispensing of prescribed drugs since July 1st, $2005 .^{22}$

Three treatment groups were built according to the severity levels based on treatment strategies. The mild group are treated with calcipotriol with or without corticosteroids (ATC: D05AX02 or D05AX52). The moderate group are treated with methotrexate (ATC: L01BA01 or L04AX03), cyclosporine (ATC: L04AD01 or L04AA01) or acitretin (ATC: D05BB02). The severe group who are treated with adalimumab (ATC: L04AB04 or L04AA17), etanercept (ATC: L04AB01 or L04AA11), infliximab (ATC: L04AB02 or L04AA12), efalizumab (ATC: L04AA21), ustekinumab (ATC: L04AC05), certolizumab (ATC: L04AB05), or golimumab (ATC: L04AB06).

To be eligible for the current study the following criteria needed to be fulfilled. First, patients require an index date (the first dispensing date in the respective treatment group, up to three possible index dates, one for each treatment group) between January 1st, 2007 and December 31st, 2013. Second, patients are at least 18 years of age at the index date. Third, patients require at least one psoriasis diagnosis (ICD-10: L40, excluding L405) within five years prior to the index date. Lastly, patients are without treatment using the same strategy, as described above, within one year prior to the index date.

In each severity group, the patients with psoriasis were followed from the first dispensing date of a certain type of psoriasis treatment (ie, the index date) to the first occurrence of the outcome, switching to a treatment strategy for a more severe psoriasis level, moving out of Sweden, death, or end of study (December 31st, 2017). Population controls were followed from the index date of their matched psoriasis patient to the first occurrence of an outcome, a psoriasis diagnosis/treatment dispensing, moving out of Sweden, death, or end of the study. In addition, patients were allowed to enter more than one severity group following the treatment hierarchy (ie, in the order of mild to moderate to severe treatments). The outcomes of the study were hypertension, hypercholesterolemia, and diabetes (see Supplementary Table 1). An illustration of the study design can be found in Supplementary Illustration 1.

\section{Statistical Analyses}

Flexible parametric models adjusted for age at index, sex, calendar year at index, and county were used to estimate the hazard ratios (HRs) of metabolic risk factors comparing patients with psoriasis with population controls. Only the first occurrence of each outcome since the index date 
was considered. Time since the index date of each psoriasis severity group was used as the underlying timescale both for patients with psoriasis and their matched population controls. Models allowing for non-proportional hazards for the effect of psoriasis were conducted to understand whether the HRs varied during follow-up, as well as models assuming proportional hazards. ${ }^{23}$ Based on the parameters from the non-proportional hazards flexible parametric models, the standardized probability (risk) for patients with psoriasis to experience metabolic outcomes was estimated at different time points following index date, based on the internal distribution of the adjusting factors within each of the severity groups separately. Similarly, the standardized risk for controls to experience metabolic outcomes was estimated. The difference of standardized risks of metabolic factors was compared between patients with psoriasis and controls and are comparable in terms of the adjusting factors. All analyses were conducted separately within each psoriasis severity group.

Data management was conducted using SAS 9.4 and all statistical analyses were conducted using STATA 15.1 package STPM2.

\section{Results}

Table 1 provides the number of patients within the mild, moderate, and severe psoriasis groups. In total, 3252 patients with psoriasis were defined as having severe psoriasis because of their hierarchical treatment exposure to biological treatment. Additionally, 14,903 patients with psoriasis were defined as having moderate psoriasis because of their hierarchical treatment exposure to nonbiological systemic treatment. Lastly, 29,434 patients with psoriasis were defined as having mild psoriasis because of their hierarchical treatment exposure to topical treatment. The patients with severe psoriasis were younger (median 49.2 years old, IQR 38.4-59.1) than the patients with moderate psoriasis (median 55.9 years old, IQR 43.865.3) or with mild psoriasis (median 55.8 years old, IQR 41.3-66.5) (Table 1). Compared to the non-psoriasis population for the respective mild, moderate, and severe psoriasis groups, the non-psoriasis population is similar for age, gender, and year of index. For example, the age distributions for the matched non-psoriasis population to the separate psoriasis severity groups are within $1-2 \%$.

The models allowing for non-proportional hazards (non-PH) in general showed that the HRs for all outcomes and within all severity groups were fairly constant over time, but with a slightly increasing $\mathrm{HR}$ early and decreasing over time (see Supplementary Figures 1-9). When testing the proportional hazard assumption, there was no evidence of non-PH, and the following results are therefore from the models assuming proportional hazards. The hazard ratio of HTN was 1.73 (95\% CI: 1.64-1.82) for patients with severe psoriasis, while it was $1.35(95 \%$ CI: 1.32-1.38) and 1.29 (95\% CI: 1.27-1.32) for patients with moderate and mild psoriasis, respectively. Regarding DM the HRs were 2.25 (95\% CI: 2.05-2.47), 1.67 (95\% CI: $1.60-1.75)$, and 1.60 (95\% CI: $1.55-1.65)$ for patients with severe, moderate and mild psoriasis, respectively. In relation to hypercholesterolemia, HR for patients with severe psoriasis was 1.58 (95\% CI: $1.47-1.70)$, while it was 1.32 (95\% CI: $1.28-1.36)$ for patients with moderate psoriasis and 1.34 (95\% CI: 1.31-1.38) for mild psoriasis (Table 2).

Table 3 demonstrates the risk of metabolic risk factors for patients with psoriasis and their matched population controls, as well as the difference in standardized risk between patients with psoriasis and their controls without psoriasis. The results show a higher standardized risk among patients with psoriasis compared with their controls in 6 months, 1 year, 5 years, and 10 years after the index date.

For example, from Table 3, patients with severe psoriasis experience a 0.58 (95\% CI: $0.56-0.59) 10$-year risk (1 minus the survival probability) of hypertension. The 10year risk for the same outcome for matched patients without psoriasis was 0.42 (95\% CI: $0.41-0.43$ ). This suggests a 0.16 (95\% CI: 0.14-0.17) increased risk of hypertension for patients with severe psoriasis compared to non-psoriasis controls.

Table 3 also demonstrates that patients with severe psoriasis experience a 0.33 (95\% CI: 0.32-0.35) 10-year risk of hypercholesterolemia. The 10-year risk for the same metabolic risk factors for matched patients without psoriasis was 0.23 (95\% CI: $0.22-0.23)$. This suggests a 0.10 (95\% CI: $0.09-0.12$ ) increased risk of hypercholesterolemia for patients with severe psoriasis compared to non-psoriasis controls.

Lastly, Table 3 shows that patients with severe psoriasis experience a 0.21 (95\% CI: $0.20-0.23)$ 10-year risk of metabolic risk factors related to diabetes. For matched patients without psoriasis, the 10 -year risk for the same outcome was 0.11 (95\% CI: $0.10-0.11)$. This suggests a 0.11 (95\% CI: 0.09-0.12) increased risk of diabetes for patients with severe psoriasis compared to non-psoriasis controls. 
Table I Baseline Characteristics of Adult Patients with Psoriasis by Severity of the Disease

\begin{tabular}{|c|c|c|c|c|c|c|}
\hline & \multicolumn{2}{|l|}{ Mild Psoriasis } & \multicolumn{2}{|c|}{ Moderate Psoriasis } & \multicolumn{2}{|c|}{ Severe Psoriasis } \\
\hline & Pso Group & Non-Pso Group & Pso Group & Non-Pso Group & Pso Group & Non-Pso Group \\
\hline Total number & 29434 & 244479 & 14903 & 123305 & 3252 & 27015 \\
\hline \multicolumn{7}{|l|}{ Sex } \\
\hline Men (\%) & I524I (5I.8\%) & $127374(52.1 \%)$ & $7785(52.2 \%)$ & $64823(52.6 \%)$ & I 764 (54.2\%) & I 4734 (54.5\%) \\
\hline \multicolumn{7}{|c|}{ Number of individuals in each age category at index } \\
\hline $\begin{array}{l}\leq 30 \\
30-40 \\
40-50 \\
50-60 \\
60-70 \\
\geq 70 \\
\text { Median age (IQR) }\end{array}$ & $\begin{array}{l}2978(10.1 \%) \\
3823(13 \%) \\
4630(15.7 \%) \\
6001(20.4 \%) \\
6664(22.6 \%) \\
5338(18.1 \%) \\
55.8(41.3-66.5)\end{array}$ & $\begin{array}{l}26775(11 \%) \\
33051(13.5 \%) \\
39246(16.1 \%) \\
48452(19.8 \%) \\
52697(21.6 \%) \\
44258(18.1 \%) \\
55(40.4-66.3)\end{array}$ & $\begin{array}{l}983(6.6 \%) \\
1767(11.9 \%) \\
2710(18.2 \%) \\
3558(23.9 \%) \\
3596(24.1 \%) \\
2289(15.4 \%) \\
55.9(43.8-65.3)\end{array}$ & $\begin{array}{l}8848(7.2 \%) \\
15276(12.4 \%) \\
22945(18.6 \%) \\
28727(23.3 \%) \\
28455(23.1 \%) \\
19054(15.5 \%) \\
55.3(43.1-65.2)\end{array}$ & $\begin{array}{l}310(9.5 \%) \\
624(19.2 \%) \\
762(23.4 \%) \\
800(24.6 \%) \\
575(17.7 \%) \\
181(5.6 \%) \\
49.2(38.4-59.1)\end{array}$ & $\begin{array}{l}2810(10.4 \%) \\
5285(19.6 \%) \\
6404(23.7 \%) \\
6451(23.9 \%) \\
4575(16.9 \%) \\
1490(5.5 \%) \\
48.5(38-58.7)\end{array}$ \\
\hline \multicolumn{7}{|c|}{ Calendar year at index } \\
\hline 2007 & II $682(39.7 \%)$ & $96663(39.5 \%)$ & $5367(36 \%)$ & $44246(35.9 \%)$ & $1035(31.8 \%)$ & $8581(31.8 \%)$ \\
\hline 2008 & $4130(14 \%)$ & $34275(14 \%)$ & 1617 (10.9\%) & 13325 (10.8\%) & 367 (II.3\%) & 3012 (11.1\%) \\
\hline 2009 & 3398 (11.5\%) & $2824 \mid(11.6 \%)$ & 1494 (I0\%) & $12313(10 \%)$ & $328(10.1 \%)$ & 2746 (I0.2\%) \\
\hline 2010 & 3080 (10.5\%) & $25478(10.4 \%)$ & 1476 (9.9\%) & I 2268 (9.9\%) & 304 (9.3\%) & 2479 (9.2\%) \\
\hline 2011 & 2568 (8.7\%) & 21366 (8.7\%) & 1633 (II\%) & 13516 (11\%) & 362 (II.1\%) & 3063 (11.3\%) \\
\hline 2012 & 2403 (8.2\%) & 20073 (8.2\%) & 1662 (II.2\%) & | 3795 (| | .2\%) & 403 (12.4\%) & 3363 (I2.4\%) \\
\hline 2013 & $2173(7.4 \%)$ & I 8383 (7.5\%) & 1654 (II.I\%) & | 3842 (| I.2\%) & 453 (13.9\%) & $377 \mid$ (14\%) \\
\hline
\end{tabular}

Overall, Table 3 demonstrates that psoriasis patients have increased risks of metabolic risk factors pertaining to hypertension, hypercholesterolemia, and diabetes relative to non-psoriasis patients. These differences between psoriasis and non-psoriasis patients increase over time and with psoriasis severity. Hazard ratios are plotted over ten years by severity for the metabolic risk factors in the Supplementary Figures.

\section{Discussion}

In this large population-based cohort study among patients with psoriasis, using the Swedish national registers, we found that the risk of metabolic risk factors related to hypertension, diabetes mellitus, and hypercholesterolemia are higher among patients with psoriasis compared with matched controls from the general population. Our findings showed that the hazard of metabolic risk factors related to hypertension, diabetes, and hypercholesterolemia is increased in line with the severity of psoriasis.

The findings of this study showed a higher risk of metabolic risk factors among patients with psoriasis, especially in severe psoriasis, which is consistent with other studies that reported the prevalence of cardiovascular risk factors in patients with mild to severe psoriasis in China and the UK. ${ }^{24,25}$ Both cited studies demonstrated that multiple cardiovascular disease risk factors are associated with psoriasis. Our study adds to this body of work by demonstrating similar conclusions from a Swedish psoriasis patient population.

Predicted risk of the metabolic risk factors in 6 months, 1 year, 5 years, and 10 years after the index date has shown a higher risk of the metabolic risk factors among patients with psoriasis compared with their matched controls. The difference between patients with psoriasis and their matched controls remains constant through ten years (see Supplementary Figures 10-18). Few studies focus on the predicted proportion of metabolic risk factors. Our findings extend the understanding of the association of metabolic risk factors and psoriasis by evaluating the long-term disease management of psoriasis compared with controls. Among the severe psoriasis patients, we found that the 10year risk of incident hypertension, hypercholesterolemia, and diabetes mellitus is $58 \%, 33 \%$, and $21 \%$, respectively. Kaye et $\mathrm{al}^{26}$ report similar measures of incident risk factors for cardiovascular diseases. The only difference between the two studies is the study population, where Kaye et al 
Table 2 Hazard Ratio (HR) and 95\% Confidence Interval of Metabolic Risk Factors Among Patients with Psoriasis Compared to Individuals Without Psoriasis

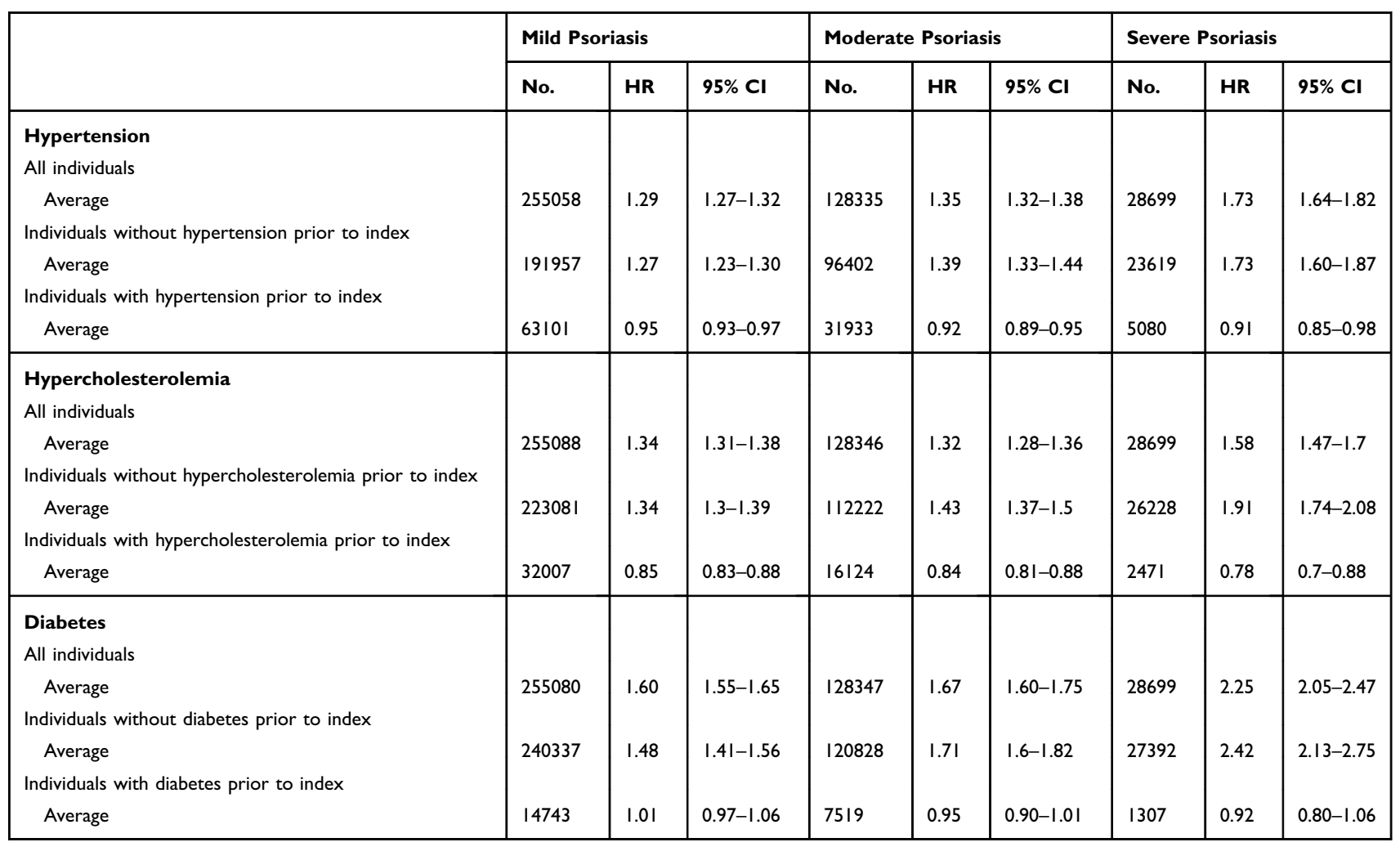

used the UK population while our study focuses on a Swedish population, appealing to the longitudinal observation period from Swedish registries. Furthermore, about $30 \%$ of the patients with psoriasis and about $20 \%$ of the controls develop more than one risk factor during the study period from index dispensing until censoring for death, emigration, or end of study (data not shown). This is possibly not a fair comparison between patients and controls if the follow-up time is different in the two groups.

One study strength is the longitudinal observation period of patients with psoriasis to pursue the research question. The nationwide coverage including all patients in Sweden with a specialist care diagnosis of psoriasis is also a major strength. The national registers allow for completeness and accuracy of the linking between them due to the unique civil registration number, given at birth or immigration and kept unchanged throughout life.

A limitation of the study is the lack of psoriasis diagnosis from primary care for patients, which may only have mild psoriasis and no contact with a specialist before the index date. The severity of psoriasis is identified via treatment strategies instead of clinical assessments due to a lack of access to any disease score. A more direct severity measure, such as PASI, is more ideal; however, these data are not captured in Swedish national health registers. Nevertheless, using anti-psoriatic treatment as a proxy for the severity of the disease has been estimated to have a sensitivity and positive predictive value of more than $90 \%$ and $80 \%$, respectively. ${ }^{27,28}$ Therefore, it is possible that the extent of the findings may occur either through the disease progression or from treatment exposure. In addition, the study uses a register-based data source; therefore, we cannot adjust for detailed health information like body mass index or laboratory biomarkers. Regarding the validity of the diagnosis of hypertension, diabetes, and hypercholesterolemia in the database we believe that diagnoses of the diseases are underreported when using hospital encounters; therefore, our disease concepts included also dispensed drugs for each disease. The treatments are included to cover diagnoses from primary care, currently not covered by the Swedish national health registers. However, the diagnoses recorded from hospital encounters are of high validity. ${ }^{20}$

\section{Conclusion}

In conclusion, our findings suggest that the hazard ratios and predicted risks of metabolic risk factors are higher 
Table 3 Risk and Risk Difference of the Metabolic Risk Factors in Patients with Psoriasis Compared to Individuals Without Psoriasis in Six Months, One Year, Five Years and 10 Years After Dispensing Date

\begin{tabular}{|c|c|c|c|c|c|c|}
\hline & \multicolumn{2}{|c|}{ Non Psoriasis } & \multicolumn{2}{|c|}{ Psoriasis } & \multicolumn{2}{|c|}{ Difference } \\
\hline & Risk & $95 \% \mathrm{Cl}$ & Risk & $95 \% \mathrm{Cl}$ & Risk & $95 \% \mathrm{Cl}$ \\
\hline \multicolumn{7}{|l|}{ Hypertension } \\
\hline \multicolumn{7}{|l|}{ Mild } \\
\hline Predicted risk at $6 \mathrm{~m}$ & 0.15 & $0.15-0.15$ & 0.17 & $0.17-0.18$ & 0.02 & $0.02-0.03$ \\
\hline Predicted risk at ly & 0.18 & $0.18-0.18$ & 0.22 & $0.21-0.22$ & 0.04 & $0.03-0.04$ \\
\hline Predicted risk at $5 y$ & 0.35 & $0.35-0.35$ & $0.4 I$ & $0.4 I-0.42$ & 0.07 & $0.06-0.07$ \\
\hline Predicted risk at $10 y$ & 0.50 & $0.50-0.50$ & 0.57 & $0.57-0.58$ & 0.07 & $0.07-0.08$ \\
\hline \multicolumn{7}{|l|}{ Moderate } \\
\hline Predicted risk at $6 \mathrm{~m}$ & 0.16 & $0.15-0.16$ & 0.18 & $0.18-0.19$ & 0.03 & $0.02-0.03$ \\
\hline Predicted risk at ly & 0.19 & $0.19-0.19$ & 0.23 & $0.22-0.23$ & 0.04 & $0.03-0.05$ \\
\hline Predicted risk at $5 y$ & 0.36 & $0.35-0.36$ & 0.44 & $0.43-0.44$ & 0.08 & $0.07-0.09$ \\
\hline Predicted risk at $10 y$ & 0.52 & $0.5 \mathrm{I}-0.52$ & 0.61 & $0.60-0.61$ & 0.09 & $0.08-0.10$ \\
\hline \multicolumn{7}{|l|}{ Severe } \\
\hline Predicted risk at $6 \mathrm{~m}$ & 0.11 & $0.11-0.12$ & 0.15 & $0.14-0.17$ & 0.04 & $0.03-0.05$ \\
\hline Predicted risk at ly & 0.14 & $0.13-0.14$ & 0.20 & $0.19-0.21$ & 0.06 & $0.05-0.07$ \\
\hline Predicted risk at $5 y$ & 0.27 & $0.27-0.28$ & 0.40 & $0.39-0.41$ & 0.13 & $0.12-0.14$ \\
\hline Predicted risk at $10 y$ & 0.42 & $0.4 I-0.43$ & 0.58 & $0.56-0.59$ & 0.16 & $0.14-0.17$ \\
\hline \multicolumn{7}{|l|}{ Hypercholesterolemia } \\
\hline \multicolumn{7}{|l|}{ Mild } \\
\hline Predicted risk at $6 \mathrm{~m}$ & 0.12 & $0.12-0.12$ & 0.15 & $0.14-0.15$ & 0.03 & $0.03-0.04$ \\
\hline Predicted risk at ly & 0.13 & $0.13-0.14$ & 0.17 & $0.17-0.18$ & 0.04 & $0.04-0.04$ \\
\hline Predicted risk at $5 y$ & 0.21 & $0.21-0.21$ & 0.27 & $0.27-0.27$ & 0.06 & $0.05-0.06$ \\
\hline Predicted risk at $10 y$ & 0.29 & $0.29-0.29$ & 0.36 & $0.35-0.37$ & 0.07 & $0.06-0.07$ \\
\hline \multicolumn{7}{|l|}{ Moderate } \\
\hline Predicted risk at $6 \mathrm{~m}$ & 0.12 & $0.12-0.12$ & 0.14 & $0.14-0.15$ & 0.02 & $0.02-0.03$ \\
\hline Predicted risk at ly & 0.14 & $0.13-0.14$ & 0.17 & $0.16-0.17$ & 0.03 & $0.03-0.04$ \\
\hline Predicted risk at $5 y$ & 0.22 & $0.21-0.22$ & 0.27 & $0.27-0.28$ & 0.06 & $0.05-0.06$ \\
\hline Predicted risk at $10 y$ & 0.30 & $0.30-0.30$ & 0.37 & $0.36-0.38$ & 0.07 & $0.06-0.08$ \\
\hline \multicolumn{7}{|l|}{ Severe } \\
\hline Predicted risk at $6 \mathrm{~m}$ & 0.08 & $0.08-0.09$ & 0.10 & $0.09-0.11$ & 0.02 & $0.01-0.03$ \\
\hline Predicted risk at ly & 0.10 & $0.09-0.10$ & 0.12 & $0.11-0.13$ & 0.03 & $0.02-0.04$ \\
\hline Predicted risk at $5 y$ & 0.16 & $0.15-0.16$ & 0.22 & $0.21-0.24$ & 0.07 & $0.06-0.08$ \\
\hline Predicted risk at $10 y$ & 0.23 & $0.22-0.23$ & 0.33 & $0.32-0.35$ & 0.10 & $0.09-0.12$ \\
\hline \multicolumn{7}{|l|}{ Diabetes } \\
\hline \multicolumn{7}{|l|}{ Mild } \\
\hline Predicted risk at $6 \mathrm{~m}$ & 0.03 & $0.03-0.03$ & 0.04 & $0.04-0.05$ & 0.02 & $0.01-0.02$ \\
\hline Predicted risk at ly & 0.04 & $0.04-0.04$ & 0.06 & $0.06-0.06$ & 0.02 & $0.02-0.03$ \\
\hline Predicted risk at $5 y$ & 0.08 & $0.08-0.08$ & 0.13 & $0.13-0.14$ & 0.05 & $0.05-0.05$ \\
\hline Predicted risk at $10 y$ & 0.13 & $0.13-0.13$ & 0.19 & $0.18-0.19$ & 0.06 & $0.06-0.07$ \\
\hline \multicolumn{7}{|l|}{ Moderate } \\
\hline Predicted risk at $6 \mathrm{~m}$ & 0.03 & $0.03-0.03$ & 0.05 & $0.04-0.05$ & 0.02 & $0.01-0.02$ \\
\hline Predicted risk at ly & 0.04 & $0.04-0.04$ & 0.06 & $0.06-0.07$ & 0.02 & $0.02-0.03$ \\
\hline Predicted risk at $5 y$ & 0.08 & $0.08-0.09$ & 0.14 & $0.13-0.14$ & 0.05 & $0.05-0.06$ \\
\hline Predicted risk at $10 y$ & 0.13 & $0.13-0.13$ & 0.20 & $0.2-0.21$ & 0.07 & $0.06-0.08$ \\
\hline \multicolumn{7}{|l|}{ Severe } \\
\hline Predicted risk at $6 \mathrm{~m}$ & 0.02 & $0.02-0.02$ & 0.04 & $0.04-0.05$ & 0.02 & $0.01-0.03$ \\
\hline Predicted risk at ly & 0.03 & $0.03-0.03$ & 0.06 & $0.05-0.07$ & 0.03 & $0.02-0.04$ \\
\hline Predicted risk at $5 y$ & 0.06 & $0.06-0.07$ & 0.14 & $0.13-0.15$ & 0.07 & $0.06-0.09$ \\
\hline Predicted risk at $10 y$ & 0.11 & $0.10-0.11$ & 0.21 & $0.20-0.23$ & 0.11 & $0.09-0.12$ \\
\hline
\end{tabular}

Abbreviations: $\mathrm{m}$, month; $y$, year. 
among patients with psoriasis compared with matched controls from the general population. This pattern is more prominent among patients with severe psoriasis.

\section{Abbreviations}

HTN, hypertension; CVD, cardiovascular diseases; DM, diabetes mellitus; HR, hazard ratio; CI, confidence interval; NPR, national patient register; PDR, prescribed drug register; CDR, cause of death register; ATC, anatomical therapeutic chemical; ICD, International Classification of Diseases.

\section{Data Sharing Statement}

This observational study is based on individual patient data from the national Swedish health registers. We are not allowed, by law, to publicly share these data. Therefore, we are not able to make these data fully available to the public.

\section{Ethical Approval and Informed Consent}

The study was approved by the Regional Ethical Review Board at Karolinska Institutet (2009/1250_31/4).

\section{Acknowledgments}

The authors like to express their appreciation to Anders Sundström, Ina Anveden-Berglind, Sean McElligott, Sverrir Valgardsson, and Nan Li for taking part in conceptualizing the study.

\section{Author Contributions}

All authors made substantial contributions to conception and design, acquisition of data, or analysis and interpretation of data; took part in drafting the article or revising it critically for important intellectual content; gave final approval of the version to be published; and agree to be accountable for all aspects of the work.

\section{Funding}

This project was funded via the public-private real-world evidence collaboration between Karolinska Institutet and Janssen Pharmaceuticals (contract: 5-63/2015).

\section{Disclosure}

M Hajiebrahimi and TML Andersson have no conflict of interest. D Hägg and M Linder are employees at CPE, which receives grants from several entities (pharmaceutical companies, regulatory authorities, contract research organizations) for the performance of drug safety and drug utilization studies. C Song and R Villacorta are employees of Janssen.

\section{References}

1. Christophers E. Psoriasis- epidemiology and clinical spectrum. Clin Exp Dermatol. 2001;26(4):314-320. doi:10.1046/j.1365-2230.2001. 00832.x

2. Parisi R, Symmons DP, Griffiths CE, Ashcroft DM. Global epidemiology of psoriasis: a systematic review of incidence and prevalence. J Clin Investig Dermatol. 2013;133(2):377-385. doi:10.1038/ jid.2012.339

3. Alexander E, Pinto J, Pal GS, Kamath N, Kuruvilla M. Disease concomitance in psoriasis: a clinical study of 61 cases. Indian $J$ Dermatol Venereol Leprol. 2001;67(2):66-68.

4. Gelfand JM, Dommasch ED, Shin DB, et al. The risk of stroke in patients with psoriasis. J Clin Investig Dermatol. 2009;129(10):24112418. doi:10.1038/jid.2009.112

5. Gelfand JM, Neimann AL, Shin DB, Wang X, Margolis DJ, Troxel AB. Risk of myocardial infarction in patients with psoriasis. JAMA. 2006;296(14):1735-1741. doi:10.1001/jama.296.14.1735

6. Henseler T, Christophers E. Disease concomitance in psoriasis. $J$ Am Acad Dermatol. 1995;32(6):982-986. doi:10.1016/0190-9622(95) 91336-X

7. Kimball AB, Guerin A, Latremouille-Viau D, et al. Coronary heart disease and stroke risk in patients with psoriasis: retrospective analysis. Am J Med. 2010;123(4):350-357. doi:10.1016/j.amjmed. 2009.08.022

8. Kimball AB, Wu Y. Cardiovascular disease and classic cardiovascular risk factors in patients with psoriasis. Int J Dermatol. 2009;48 (11):1147-1156. doi:10.1111/j.1365-4632.2009.04075.x

9. Mallbris L, Granath F, Hamsten A, Stahle M. Psoriasis is associated with lipid abnormalities at the onset of skin disease. $J$ Am Acad Dermatol. 2006;54(4):614-621. doi:10.1016/j.jaad.2005.11.1079

10. Rocha-Pereira P, Santos-Silva A, Rebelo I, Figueiredo A, Quintanilha A, Teixeira F. Dislipidemia and oxidative stress in mild and in severe psoriasis as a risk for cardiovascular disease. Clin Chim Acta. 2001;303(1-2):33-39. doi:10.1016/S0009-8981(00)00358-2

11. Shelling ML, Federman DG, Prodanovich S, Kirsner RS. Psoriasis and vascular disease: an unsolved mystery. Am J Med. 2008;121 (5):360-365. doi:10.1016/j.amjmed.2008.01.025

12. Sommer DM, Jenisch S, Suchan M, Christophers E, Weichenthal M. Increased prevalence of the metabolic syndrome in patients with moderate to severe psoriasis. Arch Dermatol Res. 2007;298(7):321. doi:10.1007/s00403-006-0703-Z

13. Mehta NN, Yu Y, Pinnelas R, et al. Attributable risk estimate of severe psoriasis on major cardiovascular events. Am J Med. 2011;124(8):775. e771-775. e776. doi:10.1016/j.amjmed.2011. 03.028

14. Lee M-S, Lin R-Y, Lai M-S. Increased risk of diabetes mellitus in relation to the severity of psoriasis, concomitant medication, and comorbidity: a nationwide population-based cohort study. $J \mathrm{Am}$ Acad Dermatol. 2014;70(4):691-698. doi:10.1016/j.jaad.2013.11.023

15. Prodanowich S, Ma F, Taylor JR, Pezon C, Fasihi T, Kirsner RS. Methotrexate reduces incidence of vascular diseases in veterans with psoriasis or rheumatoid arthritis. J Am Acad Dermatol. 2005;52 (2):262-267. doi:10.1016/j.jaad.2004.06.017

16. Wu JJ, Poon K-YT. Association of gender, tumor necrosis factor inhibitor therapy, and myocardial infarction risk in patients with psoriasis. J Am Acad Dermatol. 2013;69(4):650-651. doi:10.1016/j. jaad.2013.04.035

17. Wu JJ, Poon K-YT, Channual JC, Shen AY-J. Association between tumor necrosis factor inhibitor therapy and myocardial infarction risk in patients with psoriasis. Arch Dermatol. 2012;148(11):1244-1250. doi:10.1001/archdermatol.2012.2502 
18. Ryan C, Korman NJ, Gelfand JM, et al. Research gaps in psoriasis: opportunities for future studies. J Am Acad Dermatol. 2014;70 (1):146-167. doi:10.1016/j.jaad.2013.08.042

19. Hajiebrahimi M, Linder M, Hägg D, et al. Young patients with risk factors prevalent in the elderly-differences in comorbidity depending on severity of psoriasis: a nationwide cross-sectional study in Swedish health registers. Clin Epidemiol. 2018;10:705. doi:10.2147/ CLEP.S164918

20. Ludvigsson JF, Andersson E, Ekbom A, et al. External review and validation of the Swedish national inpatient register. BMC Public Health. 2011;11(1):1. doi:10.1186/1471-2458-11-450

21. Scocialstyrelsen. Swedish Cause of Death Register 2017.

22. Wettermark B, Hammar N, MichaelFored C, et al. The new Swedish Prescribed Drug Register-opportunities for pharmacoepidemiological research and experience from the first six months. Pharmacoepidemiol Drug Saf. 2007;16(7):726-735. doi:10.1002/ pds. 1294

23. Hultcrantz M, Björkholm M, Dickman PW, et al. Risk for arterial and venous thrombosis in patients with myeloproliferative neoplasms: a population-based cohort study. Ann Intern Med. 2018;168(5):317325. doi:10.7326/M17-0028
24. Ma L, Li M, Wang H, Li Y, Bai B. High prevalence of cardiovascular risk factors in patients with moderate or severe psoriasis in northern China. Arch Dermatol Res. 2014;306(3):247-251. doi:10.1007/ s00403-013-1437-3

25. Neimann AL, Shin DB, Wang X, Margolis DJ, Troxel AB, Gelfand JM. Prevalence of cardiovascular risk factors in patients with psoriasis. J Am Acad Dermatol. 2006;55(5):829-835. doi:10.1016/j.jaad. 2006.08.040

26. Kaye J, Li L, Jick S. Incidence of risk factors for myocardial infarction and other vascular diseases in patients with psoriasis. Br J Dermatol. 2008;159(4):895-902. doi:10.1111/j.1365-2133.2008.08707.x

27. Egeberg A, Gyldenlove M, Zachariae C, Skov L. Validation of psoriasis severity classification based on use of topical or systemic treatment. J Eur Acad Dermatol Venereol. 2018;32(1):e4-e5. doi:10.1111/jdv.14427

28. Loft N, Skov L, Bryld LE, Gislason G, Egeberg A. Treatment history of patients receiving biologic therapy for psoriasis - a Danish nationwide study. J Eur Acad Dermatol Venereol. 2017;31(8):e362-e363. doi:10.1111/jdv.14156
Clinical Epidemiology

\section{Publish your work in this journal}

Clinical Epidemiology is an international, peer-reviewed, open access, online journal focusing on disease and drug epidemiology, identification of risk factors and screening procedures to develop optimal preventative initiatives and programs. Specific topics include: diagnosis, prognosis, treatment, screening, prevention, risk factor modification, systematic reviews, risk \& safety of medical interventions, epidemiology \& biostatistical methods, and evaluation of guidelines, translational medicine, health policies \& economic evaluations. The manuscript management system is completely online and includes a very quick and fair peer-review system, which is all easy to use. 